\title{
A systematic review of short and medium-term mental health outcomes in young people
}

following sexual assault

\section{Introduction}

Sexual assault is common worldwide, peaking in mid-to-late adolescence. Global estimates for women having ever experienced non-partner sexual violence were $7.2 \%$ in 2010, with the highest rates of up to $21 \%$ of women in areas of Sub-Saharan Africa [1]. Intimate partner sexual violence against women is also common worldwide, with prevalence varying by country from $5 \%$, to up to $69 \%$ of women having ever experienced this form of sexual violence [2]. Adolescents are the group at the highest risk of sexual assault in the UK [3] with $17.8 \%$ of females aged 18-24 disclosing previous sexual abuse [4]. Figures globally appear similar, with $17.4 \%$ of females and $4.2 \%$ of males from age 1 month to 17 years experiencing sexual assault at some time in the USA [5], and $14.61 \%$ of females and $9.99 \%$ of males aged $15-17$ reporting lifetime sexual victimization in South Africa [6].

Associations between sexual abuse and adverse psychiatric outcomes have long been reported in the literature, with the strongest evidence for links with depression, post-traumatic stress disorder (PTSD), eating disorders, and suicide attempts [7-11]. However, the cross-sectional design of most studies limits the inferences that can be drawn from the results. This also makes it difficult to distinguish between the acute consequences of an index assault, lifetime psychiatric outcomes, and the progress of symptoms over time.

We conducted a comprehensive systematic review to evaluate the evidence around short and medium-term (i.e. within three years of assault) mental health outcomes in young people sexually assaulted between the ages of 10 and 24 years.

\section{Methods}

The protocol for this review was developed by clinicians and academics working in the fields of child and adolescent psychiatry, adolescent medicine, and sexual assault. The reporting of results was based on the guidelines provided by the Meta-analyses and Systematic Reviews of Observational Studies group (MOOSE guidelines) [12]. 


\section{Study question}

What are the short and medium-term effects on mental health of sexual assault between the ages of 10 and 24 years?

\section{Databases and search strategies}

Five databases (Medline (Ovid), Embase (Ovid), CINAHL (Ebscohost), OpenGrey, and PsycINFO were searched on the $30^{\text {th }}$ of October 2013 by two reviewers. This search was updated in 2016 and again in November 2018, using Medline and Embase databases only.

Key words used in the search are listed in appendix A. These were adapted as necessary for each database. The search was limited to papers having an abstract available in English; and to papers published between 1990 and the search date.

All full-text included studies and systematic reviews were searched for relevant references. Authors were contacted for any additional information if necessary in deciding whether to include or exclude the paper.

\section{Eligibility criteria}

For the purposes of this study we used the World Health Organization's definition of a 'young person' as someone aged between 10 and 24 years of age, inclusive [13]. Sexual assault was defined as any physical contact of a sexual nature taking place without the young person's consent or where the young person was unable or not deemed competent to give consent. Regarding mental health outcomes, we used the broad definition of mental disorder employed by the Centre for Disease Control (CDC): 'dysregulation of mood, thought, and/or behaviour' [14].

Inclusion criteria were as follows:

- Interventional (control arm only) or longitudinal observational studies and meta-analyses.

- Male or female participants with a mean age between 10-24 years inclusive, or where over $50 \%$ of participants fell within this age range.

- Participants must have had an initial mental health assessment occurring prior to or within 8 weeks of assault with follow-up at least 3 months after the initial assessment. 
- It was necessary to allow for a short time period post-assault within which to conduct the initial mental health assessment due to the inherent difficulties in obtaining an assessment prior to assault.

Exclusion criteria were as follows:

- Studies without an abstract available in English, case series, and conference abstracts not related to published work.

- Studies where an intervention was delivered prior to a baseline mental health assessment.

- Studies where outcomes were measured more than three years post-assault, or if there was less than three months between initial and main outcome measurements.

- The three-year time-frame was chosen to most accurately reflect short and medium-term outcomes from assaults.

\section{Study selection}

Studies were screened independently for selection by two reviewers. Any conflicting decisions were resolved by a third member of the review team. Titles were initially screened for inclusion and categorised as relevant, irrelevant, or unclear. We screened the abstracts of all studies with titles that were relevant or unclear, and then evaluated all full-text manuscripts with abstracts that were relevant or unclear. Manuscripts that could not be accessed were requested from institutional libraries, or from corresponding authors.

\section{Data extraction and quality assessment}

Data were independently extracted from included studies by two reviewers. Clarifications and additional data were sought where necessary from corresponding authors.

Quality assessment was performed independently by two reviewers using the Newcastle-Ottawa Scale (NOS) for assessing the quality of non-randomised cohort studies [15]. The scale was modified for the purpose of this review (appendix B) in order to make it more applicable to sexual assault research. An adequate follow-up retention rate was defined as $50 \%$ or more of participants being retained at the final study outcome assessment. This figure was based on average retention rates of $70 \%$ in adult sexual assault studies [16] with the hypothesis that it would be harder to retain adolescent study participants in longitudinal follow-up. We selected studies that evaluated short and medium-term mental health outcomes at least three months 
after an index assault. We considered that a three-month follow-up period would be adequate to be able to assess the trajectory of most acute psychiatric disorders.

\section{Risk of bias in individual studies}

We used the framework of the Cochrane risk of bias tool [17] to inform our assessment of selection and attrition bias only, as this tool focuses on interventional studies.

\section{Protocol and registration}

This systematic review was registered on the PROSPERO international register of systematic reviews, registration number CRD42014014023.

\section{Results}

In total, 5,124 titles and abstracts were screened after excluding duplicates, with 583 papers examined in full and ten studies selected for inclusion (Figure I). The final ten studies included in the review provided data for 984 subjects. Nine studies had prospective cohort designs. One study was a randomized controlled intervention trial that assessed reduction of sexual re-victimization rates. No meta-analyses were identified for inclusion.

[Figure I: Flowchart of study selection process]

\section{Baseline characteristics}

Of the ten studies, five included only female subjects and five included both sexes. Four studies were conducted in North America, three in Europe, and three in Sub-Saharan Africa. Eight studies recruited their participants in sexual assault referral centres (or an equivalent set-up), one study involved adolescents referred by Child Protection Services or by child abuse medical clinics, and one study was conducted with volunteer university students who reported sexual victimisation during the time of the study. (Table I).

Studies defined participants as having been subject to rape (two studies), rape or attempted rape or sexual assault (seven studies), or sexual abuse that included physical and non-physical contact (one study). Of the ten studies, seven reported on prior history of sexual 
victimization among participants, with Mouilso et al [23] only including participants presenting with a history of sexual victimization prior to the index assault. Regarding the time of the initial assessment, only Mouilso et al [23] prospectively assessed participants prior to the sexual assault. The other nine assessed participants at various time-frames post assault - within one week (Herbert et al [21]), 12 days (Rothbaum et al [27]), two weeks (Oshodi et al [26]), four weeks (Darves-Barnoz et al [18], Mutavi et al, 2017 [24], Mutavi et al, 2018 (majority of participants within one month) [25]), six weeks (Elklit et al [19], Khadr et al [22]) or within a variable timeframe ( $46 \%$ of the sample within $8-10$ weeks post-assault (Feiring et al [20]).

Studies assessed psychopathology over a minimum of three months and to a maximum of one year after the assault. (Table I)

[Table I: Characteristics of included studies]

\section{Mental health outcomes}

\section{Psychiatric Diagnosis}

Five studies evaluated whether participants met DSM criteria for psychiatric disorders at various time points (DSM III (Darves-Bornoz et al [18]), DSM III-R (Rothbaum et al [27]), and DSM IV (Elklit et al [19], Mouilso et al [23], Mutavi et al 2017 [24]). Oshodi et al [26] measured prevalence of psychiatric disorders (DSM III-R) at enrolment only. Khadr et al [22] measured prevalence of psychiatric disorders at one time point only (DSM-IV-TR).

Results in studies assessing outcomes showed very high rates of post-traumatic disorders within the first weeks of the assault (Acute Stress Disorder), which tended to decrease over the first 3-12 months. However, more than a third of victims still met PTSD criteria at final follow-up.

The overall reported PTSD rates following sexual assault were $94 \%$ within 12 days of assault , 59\% within six weeks (Acute Stress Disorder at these time-points), 65-88\% at 1 month, 10$71 \%$ at $3-4$ months, $49 \%$ at $4-5$ months, $65 \%$ at 6 months, $60 \%$ at 8 months, and $58-60 \%$ at one year (table II). 
Darves-Bornoz et al [18] found that 5\% of the sample developed delayed onset PTSD at more than 6 months post- trauma. Khadr et al [22] only looked at PTSD rates at one time point, but showed similar rates as other included studies - with $49 \%$ of participants found to have PTSD at 4-5 months post-assault. Although Mouilso et al [23] was the only study measuring PTSD rates before and after the index assault, it only included participants with a history of sexual assault. It found that $16.3 \%$ of their re-victimised student sample met criteria for PTSD four months before the sexual assault, which did not change significantly four months after the event $\left(16.3 \%\right.$ vs $\left.10.5 \%, X^{2}(1, n=86)=2.15, n s\right)$.

Of the included studies, only Darves-Bornoz et al [18] reported pre- and post-assault rates of disorders other than PTSD, reporting higher rates for most disorders post-assault (statistical comparisons not reported). However, it is not clear from the paper how these diagnoses were made. The following rates were reported pre- and 6 months post-assault, respectively: $32 \%$ and $65 \%$ for Somatoform Disorder, $34 \%$ and $44 \%$ for Social Phobia, $39 \%$ and $35 \%$ for Depressive Disorder, 10\% and 18\% for Eating Disorder, 9\% and 12\% for Psychotic or Bipolar Disorder.

Oshodi et al [26] assessed for psychiatric diagnoses at enrolment only, using a structured clinical diagnostic interview tool - the MINI-Kid (Mini International Neuropsychiatric Interview - Child and Adolescent version). Those with a known history of major psychiatric disorder were excluded prior to enrolment. At enrolment (within two weeks of rape), $22.6 \%$ of participants were diagnosed with a major depressive episode, $16 \%$ with anxiety disorder, $16.1 \%$ with adjustment disorder, and $9.7 \%$ with alcohol dependence.

Khadr et al [22] assessed for any psychiatric disorder at one time point only (4-5 months) using the DAWBA (Development and Wellbeing Assessment) - a structured diagnostic interview based on DSM-IV criteria. They found high rates; with $79 \%$ of all participants found to be suffering from any psychiatric disorder. Prevalence was highest for PTSD (49\%), any depressive disorder (43\%), conduct disorder (22\%), and generalised anxiety disorder (18\%). $55 \%$ of participants had multiple (defined as two or more) disorders.

\section{Psychiatric symptoms}

Post-traumatic stress symptoms: 
Mouilso et al [23], Feiring et al [20], Herbert et al [21], Oshodi et al [26], and Khadr et al [22] measured the presence of post-traumatic stress symptoms following sexual assault, with follow-up times of between three months and one year. In all studies, mean post-traumatic symptom scores decreased over the follow-up periods (table II). Mouilso et al [23] was the only study that compared post-traumatic symptoms before and after the assault, finding a decrease between mean scores prior to the assault and at four-months post-assault. However, it is of note that no statistically significant $p$ values were reported, and that this was the only study where all participants had experienced previous sexual assault, and were exclusively University students.

Depressive symptoms:

Five studies [20-22, 25-26] measured the presence of depressive symptoms following sexual assault. Feiring, Herbert, Khadr and Mutavi found decreasing scores over the follow-up periods (table II). Herbert et al [21] found a statistically significant decrease during the first three months but there was no further significant change by six months post-assault. Feiring et al [20] found a statistically significant decrease at 1 year after the trauma compared to symptoms at two months after the trauma. Khadr et al [22] found a statistically significant decrease in symptom score over their 4-5 month follow-up period (female participants only compared). Mutavi et al 2018 [25] did not state symptom scores but rather number of participants in each symptom category (minimal, mild, moderate or severe symptoms). Over their 1 year follow-up period, they found that for both those under 16 (Children's Depression Inventory used), and those over 16 (Becks Depression Inventory used), the trend was for a reduction in symptom scores. Among both age groups, the majority of participants reported moderate depressive symptoms at baseline, $(74.3 \%$ and $57.4 \%$ of participants for under $16 \mathrm{~s}$ and over $16 \mathrm{~s}$ respectively), shifting to a majority reporting mild depressive symptoms at 1 year follow-up (100\% and 79.4\% respectively). Oshodi et al [26] showed a very slight increase in depressive symptomatology from initial assessments to three months, which then plateaued out and maintained over the year of follow-up.

\section{Anxiety symptoms:}

Three studies looked at anxiety symptoms. Herbert et al [21] measured the presence of anxiety-related symptoms, finding high scores at initial assessment which then decreased over the 6 months post-assault. The difference was only significant between baseline and three months (table II). Oshodi et al [26] measured the presence of anxiety related symptoms, categorising these using the Multidimensional Anxiety Scale for Children (MACK) into harm avoidance, 
physical symptoms, separation anxiety, and social anxiety. For all but social anxiety (where scores were not significantly different at any time point), anxiety symptoms reduced between baseline and three months and then stabilised over time. Khadr et al [22] found a reduction in median anxiety symptom scores over their 4-5 month follow-up period, however the difference in scores was not statistically significant (on comparing female participants only).

Suicidal behaviour:

Only Darves-Bornoz et al [18] assessed suicide attempts. At study entry, 24\% of the sample reported a prior history of suicide attempts, with $22 \%$ of the sample reporting a suicide attempt at some point during the 6 months following the assault. Mouilso et al [23] and Oshodi et al [26] excluded those reporting suicidal ideation prior to enrolment, however Oshodi et al [26] on conducting the MINI-Kid interview at baseline (within 2 weeks of rape), found $54.8 \%$ of participants to have a 'suicide current risk'.

[Table II: Main mental health outcomes after sexual assault by included studies]

\section{Quality assessment}

Each study was assessed for quality using the Newcastle Ottawa Scale (NOS) for cohort studies (table III). Scores were awarded with a maximum possible score of nine stars. Mouilso et al [23] and Herbert et al [21] scored three stars. Elklit et al [19], Feiring et al [20], Mutavi et al 2017 [24], Mutavi et al 2018 [25], and Oshodi et al [26] scored four stars. Darves-Bornoz et al [18], Rothbaum et al [27] and Khadr et al [22] scored five stars.

\section{Selection}

As shown in Table III, nine studies assessed only those victims that sought help in relation to the assault (categorised as somewhat representative), with the Mouilso et al study [23] using a group of previously victimized student volunteers (selected group). This was also the only study that assessed the victims prior to the index sexual assault and that demonstrated whether the main outcome of interest was present at start of study. Darves-Bornoz et al [18] reported retrospective data on the presence of several psychiatric disorders prior to the assault, but not for PTSD, which was the main outcome. At baseline Khadr et al [22] used the Strength and Difficulties Questionnaire to provide a retrospective measurement of general psychological difficulties in the previous six months (i.e. prior to the index assault). They 
also reported on the percentage of participants seeking mental health help in the 12 months before the assault.

\section{Comparability}

No study designs included a non-exposed cohort to compare with the sexually assaulted cohort. No studies controlled for prior psychopathology or sexual victimisation. Therefore, no studies were given any stars on the bases of their design or analyses within the comparability section of the NOS. Only Elklit et al [19] analysed those that had reported prior assault separately to those for whom the index assault was their first experience of sexual assault.

\section{Outcome}

Darves-Bornoz et al [18] and Rothbaum et al [27] used clinician-led structured diagnostic interviews to measure mental health outcomes at multiple time points. Khadr et al [22] used structured interviews at one time-point and questionnaires at multiple time-points. The remaining studies used self-reported measures. Herbert et al [20] was the only study where retention rates were less than 50\%. Follow-up periods were long enough for the outcomes to occur in all studies but the Mouilso et al study [23], where the exact time between the assault and the assessment of the outcome was not specified (range 0-4 months).

[Table III: Quality assessment of included studies]

\section{Risk of bias}

Selection bias

No study had an exposed cohort that was truly representative of the average sexual assault victim in the community; nine studies [18-22, 24-27] only selected those participants who had sought help from a specialist centre, or come to the attention of authorities. Mouilso et al [23] recruited participants from a US university, who received both course credit and monetary compensation. Those screening positive for a past history of unwanted sexual contact were included, and only participants who reported being re-victimised during a 4-month follow-up period were analysed. However, it is not clear whether students were aware of the nature of the study prior to recruitment. Elklit et al [19] was the only other study to have 
controlled for the young person having experienced a prior assault as it analysed both groups separately (however the full results were reported for all participants only).

\section{Attrition bias}

Rates of those lost to follow-up were highly variable between all included studies, ranging from 0 to $61 \%$ by study end-points. Darves-Bornoz et al [18], Herbert et al [21], and Rothbaum et al [27] considered differences in socio-demographic characteristics, nature of the sexual assault and past psychiatric symptoms between lost and tracked cases. Herbert et al [21] found that depressive and anxiety symptoms in the acute aftermath of the trauma did not differ between those retained and those lost at six-months follow-up. Those with lower initial post-traumatic symptoms were found to be more likely to drop out of the study. Darves-Bornoz et al [18] reported no significant differences in PTSD rates in those who dropped out and those who remained in the cohort even though there was a slight trend to a lower frequency of pathology in those lost at follow-up.

\section{Funding}

All included studies reported on sources of funding.

\section{Discussion}

This systematic review found high levels of PTSD in young people up to a year following sexual assault. Anxiety symptoms were highest in the immediate aftermath of the trauma and generally reduced over time.

Depressive symptomology appeared to vary between studies. Four of the five studies measuring this outcome $[20-22,25]$ showed similar patterns of symptoms decreasing over time. However, Oshodi et al [26] showed persistent depressive symptoms over time. The difference could be in part related to the small number of participants in the Oshodi study (the lowest of all included studies), and to its high initial rates of depressive disorder (22.6\% met criteria for a major depressive episode at enrolment).

While levels of depressive and anxiety symptoms remained raised at most study end-points, mean scores did not indicate high risk for depressive or anxiety disorders, except for in the Oshodi et al study [26], when compared to population norms [28-32] 
According to national US surveys, the lifetime prevalence of PTSD for adult women is $9.7 \%$, and the six-month prevalence for adolescent girls is $6.3 \%[33,34]$. The risk of developing PTSD is higher in victims of sexual trauma [35], with the National Women's Study conducted in 1990 in the US finding that nearly one-third of rape victims developed PTSD [36]. Indeed, four of the five studies examining PTSD rates in sexually victimised youths [18-19, 24,27 found rates more than five times higher than those found in the general population, even by study end-points. However, when interpreting these results it is necessary to consider that the participants included in this review (i.e. victims seeking help after the assault or university students) may not be representative of the general assaulted young population. Only the study that used a non-clinical sample reported PTSD rates that resemble those estimated in the general population (Mouilso et al [23]). We believe that the variation between the four studies with high rates of PTSD [18-19, 24,27], and the fifth study with low rates of PTSD (Mouilso et al [23]), could be related to both the difference in methods of diagnosis between the five studies, and the difference in source of participants (sexual assault centre in the studies with higher rates, versus self-selected non-clinical participants). In the Mouliso study [23], the lower rates of PTSD could be linked to this sample representing victims who are likely to be higher functioning individuals, by nature of still being enroled in college following an assault, implying a lower level of morbidity than other victims. This study also excluded those with suicidal ideation at baseline, again increasing the likelihood that this sample represented less distressed victims. Since it has been shown that many victims do not report or seek help after sexual assault [37], true PTSD rates in assaulted victims from the general population remain unclear. Of note, within four weeks of trauma, the diagnosis of PTSD as it is used in some included studies, would now be referred to as acute stress disorder.

The results of this review are limited by the low number of included studies, the small sample sizes evaluated and the strict inclusion criteria. Good quality, large, and prospective studies in the field of sexual assault and youth mental health are lacking.

Comparability between studies was also limited as studies were largely heterogeneous in regards to definitions of sexual assault, recruitment methods, sample characteristics, timing of assessments and measures used to assess mental health outcomes. The majority of included studies pertained to those in the later stages of adolescence. Due to the paucity of studies focusing exclusively in the study age range, studies where over fifty percent of participants met the defined adolescent age range were included. 
Only three studies assessed for the presence of disorder at multiple time-points using operationalised criteria (Darves-Bornoz et al [18] Herbert et al [21], Rothbaum et al [27]). Furthermore, the ecological validity was limited to specific groups of victims and a narrow range of psychopathology. There is a need for further longitudinal research assessing a wider range of psychiatric difficulties, including the presence of comorbidity, neurodevelopmental disorders and externalising disorders. Only half of the included studies [19,22,24-26] reported on participants receiving any form of mental health interventions during the study time-frame.

The difficulties in conducting a baseline assessment pre-sexual assault and the lack of nonexposed control groups further limit the scope of the review, since this precludes calculating the relative risk and incidence of new onset psychopathology in relation to sexual assault. This prevents assessment of whether young people with mental health disorders are more vulnerable to assault, whether mental health disorders result from sexual assault, or whether there is bidirectional causality. Khadr et al [22] participants (therefore limited to those presenting to a sexual assault centre and agreeing to participate in a research study) were found to have very high levels of deprivation and pre-existing vulnerability (including living with only one parent, having a statement of educational needs, previous involvement with social services, accessing mental health help). Although this information was collected retrospectively, a number of these vulnerability factors were found to be associated with occurrence of any psychiatric disorder post-assault, supporting the bidirectional causality theory.

This review was conducted using sound methodology, including the use of strict inclusion criteria, considering only studies with longitudinal designs. We followed established guidelines and validated quality assessment tools that pertain particularly to systematic reviews of observational studies. These helped to ensure that included studies were of the highest quality possible from the pool of available literature. Relaxation of the inclusion criteria would have reduced the quality of included studies and therefore any conclusions drawn, but would have allowed for a larger sample size and perhaps a better overview of the range of mental health outcomes associated with sexual assault.

A considerable proportion of young people had persistent symptoms after sexual assault. Clinicians have an important role in identifying those requiring specialist intervention. Management guidelines for PTSD in the UK [38] indicate that the first line intervention should be trauma-focused psychological treatment for those with higher initial symptoms. However, clinicians have concerns regarding when and how best to start exposure therapies [39], which 
have also been associated with disengagement and symptom exacerbation [40]. There is a need for further research on the wide range of psychiatric disorders that may arise during the acute phases after a sexual trauma, which would guide on how to best focus interventions in these early stages.

\section{Conclusions}

Sexual assault remains common in the UK and globally, particularly affecting females in the adolescent age group. This review demonstrates the paucity of high quality prospective data following a significant and very prevalent event. The results show high levels of PTSD, anxiety and depressive symptoms following sexual assault in young people. Prospective studies using representative samples and robust measures for psychopathology are urgently required.

\section{References}

[1] Abrahams N, Devries K, Watts C, et al. Worldwide prevalence of non-partner sexual violence: a systematic review. Lancet. 2014 May 10;383(9929):1648-1654. doi: 10.1016/S01406736(13)62243-6.

[2] WHO multi-country study on women's health and domestic violence against women: summary report of initial results on prevalence, health outcomes and women's responses. Geneva, World Health Organization, 2005. Available at URL: https://apps.who.int/iris/bit-

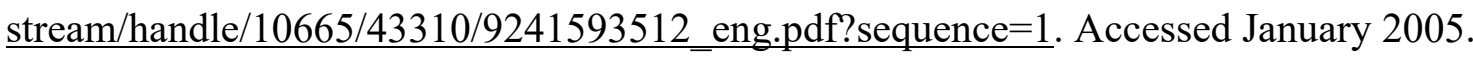

[3] Ministry of Justice, Home Office \& the Office for National Statistics. An overview of sexual offending in England and Wales. Statistics bulletin, 2013. Available at: https://www.gov.uk/government/statistics/an-overview-of-sexual-offending-in-england-andwales. Accessed July 19, 2017.

[4] NSPCC. Child abuse and neglect in the UK today. Available at: https://www.nspcc.org.uk/globalassets/documents/research-reports/child-abuse-neglect-uktoday-research-report.pdf. Accessed July 19, 2017. 
[5] Finkelhor D, Turner HA, Shattuck A, et al. Violence, Crime, and Abuse Exposure in a National Sample of Children and Youth. An Update. JAMA Pediatr. 2013;167(7):614-21. doi:10.1001/jamapediatrics.2013.42.

[6] Ward CL, Artz L, Leoschut L, et al. Sexual violence against children in South Africa: a nationally representative cross-sectional study of prevalence and correlates. Lancet Glob Health. 2018 Apr;6(4):e460-e468. doi: 10.1016/S2214-109X(18)30060-3.

[7] Chen LP, Murad MH, Paras ML, et al. Sexual Abuse and Lifetime Diagnosis of Psychiatric Disorders: Systematic Review and Meta-analysis. Mayo Clinic Proceedings 2010;85(7):618-29. doi: 10.4065/mcp.2009.0583.

[8] Gilbert R, Widom CS, Browne K, et al. Burden and consequences of child maltreatment in high-income countries. Lancet 2009;373: 68-81. doi: 10.1016/S0140-6736(08)61706-7.

[9] Jumper SA. A meta-analysis of the relationship of child sexual abuse to adult psychological adjustment. Child Abuse Negl. 1995;19(6): 715-28. doi: https://doi.org/10.1016/01452134(95)00029-8.

[10] Paolucci EO, Genuis ML, Violato C. A meta-analysis of the published research on the effects of child sexual abuse. J Psychol. 2001 135:17-36. doi:

http://dx.doi.org/10.1080/00223980109603677.

[11] Smolak L, Murnen SK. A meta-analytic examination of the relationship between child sexual abuse and eating disorders Int J Eat Disord. 2002;31(2)136-150. doi: http://dx.doi.org/10.1002/eat.10008.

[12] Stroup DF, Berlin JA, Morton SC, et al. Meta-analysis of observational studies in epidemiology: a proposal for reporting. Meta-analysis of Observational Studies in Epidemiology (MOOSE) group. JAMA 2000;283(15): 2008-12. doi: doi:10.1001/jama.283.15.2008.

[13] World Health Organization, $64^{\text {th }}$ World Health Assembly, Youth and Health Risks, May 2011. Available at:

http://www.who.int/hac/events/wha_a64_r28_en_youth_and_health_risks.pdf. Accessed July 19, 2017. 
[14] Centers for Disease Control and Prevention, Mental Health, Mental Illness. Available at: http://www.cdc.gov/mentalhealth/basics/mental-illness.htm. Accessed January 14, 2015.

[15] Wells GA, Shea B, O'Connell D, et al. Ottawa Hospital Research Institute. The Newcastle-Ottawa Scale (NOS) for assessing the quality of nonrandomised studies in meta-analyses. Available at: http://www.ohri.ca/programs/clinical_epidemiology/oxford.asp Accessed December 12, 2014.

[16] Campbell R, Brown Sprague H, Cottrill S, et al. Longitudinal research with sexual assault survivors: a methodological review. Journal of Interpersonal Violence 2011;26(3): 43361. doi: https://doi.org/10.1177/0886260510363424.

[17] Higgins JPT, Altman DG, Gøtzsche PC, et al. The Cochrane Collaboration's tool for assessing risk of bias in randomised trials $B M J$ 2011;343:d5928. doi:

https://doi.org/10.1136/bmj.d5928

[18] Darves-Bornoz JM, Lepine JP, Choquet M, et al. Predictive factors of chronic post-traumatic stress disorder in rape victims. Eur Psychiatry 1998;13:281-87. doi: 10.1016/S09249338(98)80045-X.

[19] Elklit A, Christiansen DM. ASD and PTSD in rape victims. J Interpers Violence. 2010 Aug;25(8):1470-88. doi: 10.1177/0886260509354587.

[20] Feiring C, Taska L, Lewis M. Adjustment following sexual abuse discovery: the role of shame and attributional style. Dev Psychol. 2002;38(1): 79-92. doi: 10.1037//00121649.38.1.79.

[21] Herbert CP, Grams GD, Berkowitz J. Sexual assault tracking study: who gets lost to follow-up? Can Med Assoc J. 1992;147(8):1177-84.

[22] Khadr S, Clarke V, Wellings K, Villalta L, Goddard A, Welch J, Bewley S, Kramer T, Viner R. Mental and sexual health outcomes following sexual assault in adolescents: a prospective cohort study. Lancet Child Adolesc Health. 2018 Sep;2(9):654-665. doi: 10.1016/S2352-4642(18)30202-5. 
[23] Mouilso ER, Calhoun KS, Gidycz CA. Effect of participation in a sexual assault risk reduction program on psychological distress following revictimization. J Interpers Violence 2011;26(4):769-88. doi: https://doi.org/10.1177/0886260510365862.

[24] Mutavi T, Mathai M, Obondo A. Post-Traumatic Stress Disorder (PTSD) in Sexually Abused Children and Educational Status in Kenya: A Longitudinal Study. $J$ Child Adolesc Behav. 2017;5(5). pii: 357. doi: 10.4172/2375-4494.1000357.

[25] Mutavi, T., Obondo, A., Kokonya, D., Khasakhala, L., Mbwayo, A., Njiri, F., \& Mathai, M. (2018). Incidence of depressive symptoms among sexually abused children in Kenya. Child and Adolescent Psychiatry and Mental Health, 12, 40. https://doi.org/https://dx.doi.org/10.1186/s13034-018-0247-y

[26] Oshodi Y, Macharia M, Lachman A, et al. Immediate and Long-Term Mental Health Outcomes in Adolescent Female Rape Survivors. J Interpers Violence. 2016 Dec 1:886260516682522. doi: 10.1177/0886260516682522.

[27] Rothbaum BA, Foa EB, Riggs DS, et al. A prospective examination of post-traumatic stress disorder in rape victims. Journal of Traumatic Stress 1992;5(3): 455-75. doi: https://doi.org/10.1007/BF00977239.

[28] Foa, E. Posttraumatic Diagnostic Scale Manual. Minneapolis, MN: National Computer Systems, 1996.

[29] Sheeran T, Zimmerman, M. Screening for posttraumatic stress disorder in a general psychiatric outpatient setting. Journal of Consulting and Clinical Psychology, 2002;70(4): 961-6. doi: http://dx.doi.org/10.1037/0022-006X.70.4.961

[30] Beck AT, Steer RA, Garbin MG. Psychometric properties of the Beck Depression Inventory Twenty-five years of evaluation. Clin. Psych. Review, 1988;8:77-100. doi: 10.1016/0272-7358(88)90050-5 
[31] Kovacs M. The Children's Depression Inventory (CDI) Manual. North Tonawanda, NY: Multi-Health Systems, Inc, 1992.

[32] Spielberger CD. Manual for the State-Trait Anxiety Inventory (Form Y). Palo Alto, CA: Consulting Psychologists Press, 1983.

[33] National Comorbidity Survey. (2005). NCS-R appendix tables: Table 1. Lifetime prevalence of DSM-IV/WMH-CIDI disorders by sex and cohort. Table 2. Twelve-month prevalence of DSM-IV/WMH-CIDI disorders by sex and cohort. Available at: http://www.hcp.med.harvard.edu/ncs/publications.php. Accessed July 19, 2017.

[34] Kilpatrick DG, Ruggiero KJ, Acierno R, et al. Violence and risk of PTSD, major depression, substance abuse/dependence, and comorbidity: results from the National Survey of Adolescents. Journal of Consulting and Clinical Psychology 2003;71(4):692-700.

doi: 10.1037/0022-006X.71.4.692.

[35] Cuffe SP, Addy CL, Garrison CZ, et al. Prevalence of PTSD in a Community Sample of Older Adolescents. J Am Acad Child Adoles Psychiatry 1998;37(2):147-54. doi: 10.1097/00004583-199802000-00006.

[36] Kilpatrick DG, Edmunds C, Seymour A. Rape in America: A Report to the Nation. Arlington, VA: National Victims Center, 1992

[37] Macdowall W., Gibson LJ., Tanton C., et al. (2013) Lifetime prevalence, associated factors, and circumstances of non-volitional sex in women and men in Britain: findings from the third National Survey of Sexual Attitudes and Lifestyles (Natsal-3). Lancet 382 (9907): 1845 55 doi: 10.1016/S0140-6736(13)62300-4

[38] NICE Post-traumatic stress disorder. NICE guideline [NG116] Published date: December 2018. Available at URL: https://www.nice.org.uk/guidance/ng116 . Accessed January 2019.

[39] Jaycox LH, Foa EB. Obstacles in implementing exposure therapy for PTSD: case discussions and practical solutions. Clin Psychol Psychother. 1996;3:176-184

doi: 10.1002/(SICI)1099-0879(199609)3:3<176::AID-CPP100>3.0.CO;2-1 
[40] Pitman RK, Altman B, Greenwald E, et al. Psychiatric complications during flooding therapy for posttraumatic stress disorder. J Clin Psychiatry 1991; 52:17-20. 


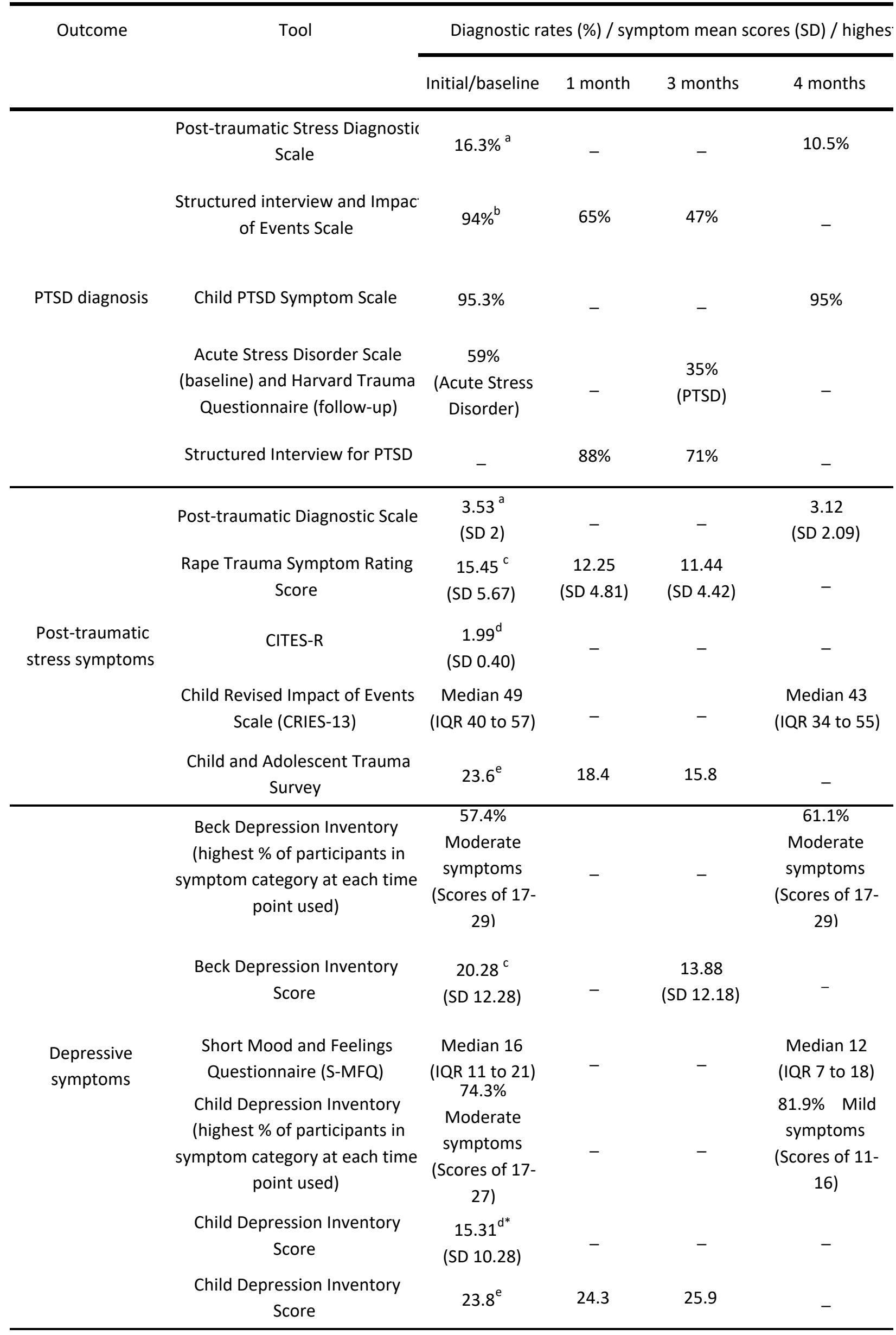




\begin{tabular}{|c|c|c|c|c|c|}
\hline $\begin{array}{l}\text { State and } \\
\text { Trait }\end{array}$ & $\begin{array}{l}\text { State Anxiety } \\
\text { Index }\end{array}$ & $\begin{array}{c}56.55^{\mathrm{c}} \\
(\mathrm{SD} 14.52)\end{array}$ & - & $\begin{array}{c}44.46 \\
\text { (SD 16.42) }\end{array}$ & - \\
\hline \multirow[t]{2}{*}{$\begin{array}{l}\text { Anxiety } \\
\text { Index }\end{array}$} & Trait Anxiety Index & $\begin{array}{c}49.66^{\mathrm{C}} \\
(\mathrm{SD} 12.54)\end{array}$ & - & $\begin{array}{c}45.14 \\
\text { (SD 15.93) }\end{array}$ & - \\
\hline & Harm Avoidance & $11.5^{\mathrm{e}}$ & 10.3 & 10.5 & - \\
\hline \multirow{3}{*}{$\begin{array}{l}\text { Multi- } \\
\text { dimensional } \\
\text { Anxiety Scale } \\
\text { for Children }\end{array}$} & Physical symptoms & $15.8^{e}$ & 12.7 & 11.2 & - \\
\hline & Separation Anxiety & $14.35^{\mathrm{e}}$ & 10.8 & 9.8 & - \\
\hline & Social Anxiety & $17.1^{\mathrm{e}}$ & 16.5 & 16.8 & - \\
\hline \multicolumn{2}{|c|}{$\begin{array}{l}\text { The Screen for Child Anxiety } \\
\text { Related Disorder (SCARED) }\end{array}$} & $\begin{array}{c}\text { Median } 37 \\
\text { (IQR } 28 \text { to } 52 \text { ) }\end{array}$ & - & - & $\begin{array}{c}\text { Median } 34 \\
\text { (IQR } 22 \text { to } 47 \text { ) }\end{array}$ \\
\hline
\end{tabular}

\footnotetext{
${ }^{\mathrm{a}}$ baseline at 0-4 month prior sexual assault, ${ }^{\mathrm{b}}$ baseline at 12 days after sexual assault, ${ }^{\mathrm{c}}$ baseline within 1 week $\mathrm{SD}=$ Standard Deviation, PTSD = Post-Traumatic Stress Disorder

Note. Dashes indicate outcome not assessed at that time point

* - figures stated relate to female participants only
} 


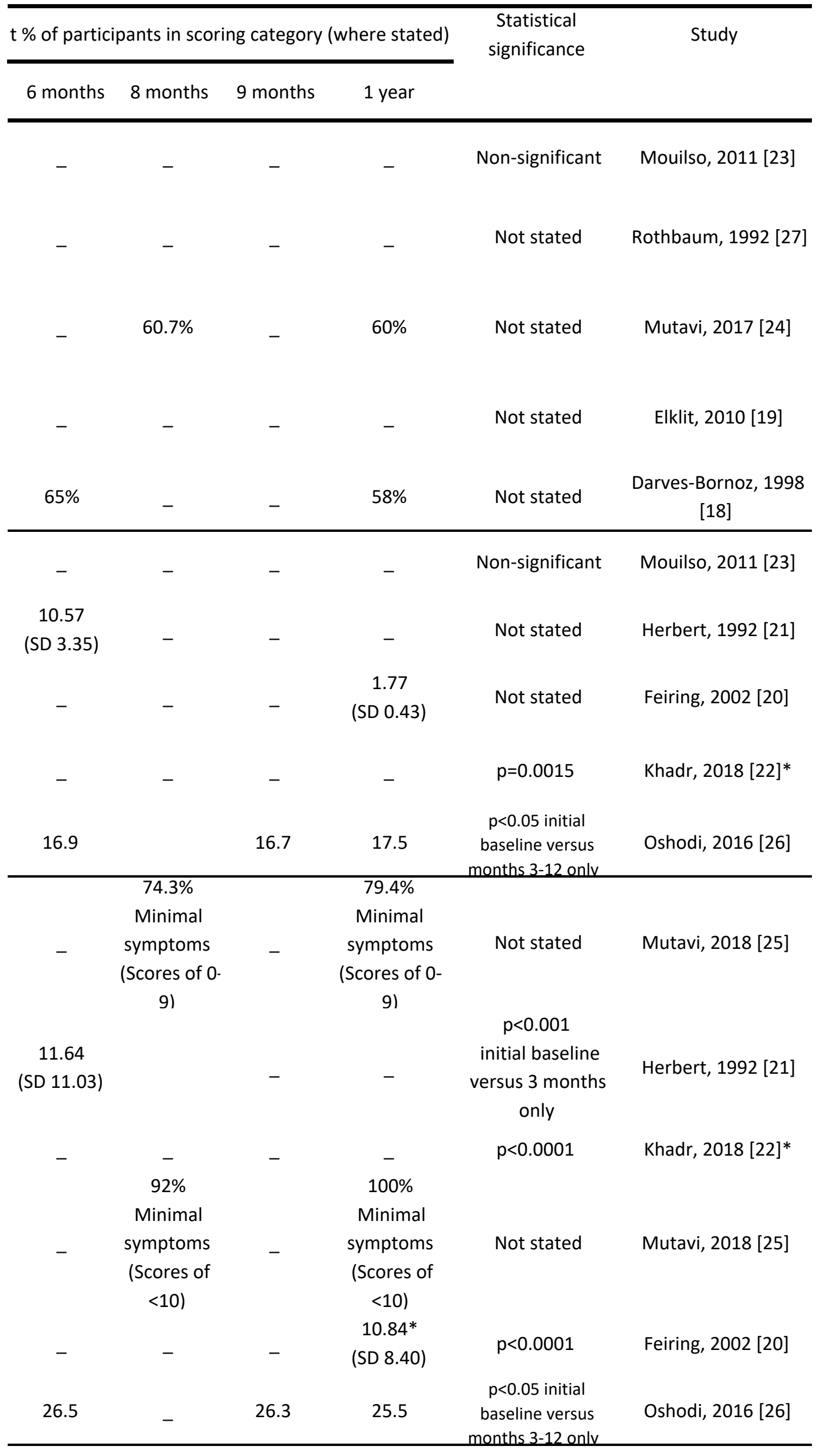


43.14

(SD 14.18)

45.22

(SD 13.91)

8.9

10.7

9

16.4

p $<0.001$ initial
baseline versus 3
months only
$p<0.001$ initial
baseline versus 3
months only
$p<0.05$ initial
baseline versus
months 3-12 only
$p<0.05$ initial
baseline versus
months 3-12 only
$p<0.05$ baseline
versus all
timepoints

of sexual assault, ${ }^{\mathrm{d}}$ baseline at 8-10weeks after discovery of sexual assault, ${ }^{\mathrm{e}}$ baseline within 2 weeks of $\mathrm{r}$; 

